\title{
Case study-based time-course analysis of symptoms of respiratory syncytial virus infections followed by acute sinusitis in otherwise-healthy adults
}

\author{
Hidekazu Nishimura ${ }^{1}$, Ko Sato ${ }^{1}$, Francois Marie Ngako Kadji ${ }^{1}$, Suguru Ohmiya ${ }^{1}$, Hiroko Ito ${ }^{1}$, Toru \\ Kubo $^{1,3,4}$, Sho Hashimoto ${ }^{2}$ \\ ${ }^{1}$ Virus Research Center, Clinical Research Division, ${ }^{2}$ Department of Otolaryngology, Sendai Medical Center, National Hospital Organization, \\ Sendai, Miyagi, Japan; ${ }^{3}$ Department of Virology, Institute of Tropical Medicine, Nagasaki University, Nagasaki, Japan; ${ }^{4}$ Nagasaki Genbaku Isahaya \\ Hospital, Isahaya, Nagasaki, Japan \\ Correspondence to: Hidekazu Nishimura. Virus Research Center, Clinical Research Division, Sendai Medical Center, National Hospital Organization, \\ Sendai, Miyagi, Japan. Email: hide-nishimura@mte.biglobe.ne.jp.
}

\begin{abstract}
Reports on respiratory syncytial virus (RSV) infections are abundant in pediatric and geriatric populations but not many in healthy adults, and particularly, those which demonstrated the illness throughout its time course are rare. We report two otherwise-healthy adult cases, showing a number of evidence essential for confirmation of exclusive infections with RSV, and document their clinical features from the onset of the disease to recovery, including secondary sinusitis with magnetic resonance (MR) and computed tomography (CT) images. The infection was proven by isolating RSV belonging to subgroup $\mathrm{B}$ and by observing elevated anti-RSV antibody titer in the paired sera. Possible contribution of other pathogens including almost all respiratory viruses and representative bacteria, was excluded by negative results in multiplex PCR examination. In the first case, illness initiated with pharyngeal pain, followed by symptoms of sneezing, severe rhinorrhea and coughing, which peaked at approximately 5-7 days and persisted for 12 days. The patient experienced a slight chill, but the body temperature did not exceed $37{ }^{\circ} \mathrm{C}$ during illness. The patient showed no significant finding but only a slight increase in serum C-reactive protein level in the routine clinical laboratory examinations. On the 9th day of illness, a dull headache started persisting for at least a week after which it gradually waned. Sinusitis was found by chance on MR images of maxillary sinus 8 days after the headache started, and the finding disappeared on CT images taken after 6 months. In the second case, the symptoms included severe rhinorrhea and dull facial pain around the upper nose; the pain also occurred on the 9th day of illness and the symptom was clinically diagnosed to be acute sinusitis during a visit to a physician.
\end{abstract}

Keywords: Respiratory syncytial virus (RSV); healthy adult case; rhinorrhea; acute sinusitis; magnetic resonance imaging (MRI)

Submitted Aug 22, 2017. Accepted for publication Apr 09, 2018.

doi: $10.21037 /$ jtd.2018.04.74

View this article at: http://dx.doi.org/10.21037/jtd.2018.04.74

\section{Introduction}

Human respiratory syncytial virus (RSV) is a single stranded RNA virus, a member of the family Pneumoviridae and genus Orthopneumovirus, and comprises two subgroups, A and B. Many studies have been published world wide regarding clinical features of RSV infection in pediatric, elderly, and immune-compromised adult populations in detail to demonstrate the clinical significance of the infection (1). In healthy adult population, several pioneering studies were performed from the late 1970's to early 2000's (2) and, thereafter, studies on healthy young adults of military trainees were performed (3-5), and they showed that 


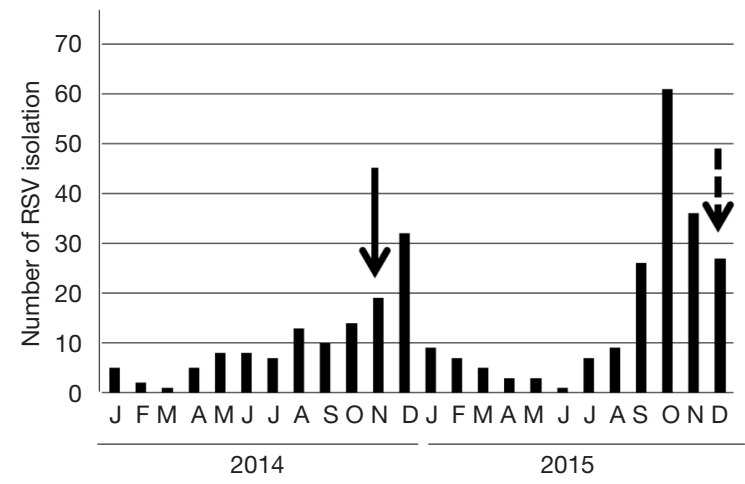

Figure $1 \mathrm{RSV}$ isolation by a reference laboratory in Sendai city in 2014 and 2015. Isolating RSV from pediatric patients with acute respiratory diseases in Virus Research Center, Sendai Medical Center. The timing of the RSV from the first case: bold arrow indicates the day when RSV was isolated from the first case; dotted line with arrow denote the day when RSV was isolated from the second case. RSV, respiratory syncytial virus.

the infection was clinically significant in those population, as well. However, their main data were about the symptoms and their durations, and there has been a lack of detailed information, particularly regarding the progression of infection from the onset of the illness. Exceptions to this were the reports of experimental infections where a relatively high dose of virus was administered by pouring viral fluid drops into the nasal cavity $(6,7)$. However, in these reports, the available information only concerned the infection, the description of clinical features was very simple, and the time points of the observations were unclear and did not follow a time course. In this study, we report two cases of healthy adults with community-acquired RSV infections, which were virologically proven to be a single infection without any contribution from other pathogens. In these cases, we systematically recorded the clinical features in detail, from the onset to end of the illness through to acute sinusitis diagnosis. We report a case showing computed tomography (CT) and magnetic resonance (MR) images, which were taken for other reasons and revealed the sinusitis by chance. We further introduce an additional case, which was also a single RSV infection, and showing almost the same clinical features as those of the first case, including the symptom, which was suggestive of acute sinusitis.

\section{Case presentation}

The case was a 59-year-old male living in Sendai City,
Japan, who did not have any health problems related to infectious diseases. On November 5, 2014, he developed a sore throat without any specific findings on the pharyngeal wall. During this time, the number of children infected with RSV was increasing in the city, leading to an RSV infection epidemic (Figure 1). On the following day, slight fever, fatigue, and chillness occurred. In addition, his body temperature slightly increased to approximately $37{ }^{\circ} \mathrm{C}$, whereas his usual body temperature ranged between 35.8 and $36.2{ }^{\circ} \mathrm{C}$. Two days after the onset of illness (day of illness 2: DOI 2), symptoms of sneezing and rhinorrhea started. Rhinorrhea became severe with continuous running nose, and coughing started on DOI 3. The cough persisted for 12 days and peaked at approximately DOI 5-7. Clear and relatively serous sputum was coughed up at the time when the symptom of cough initiated. On the other hand, the mucus of the sputum coughed up 1 week later was sticky and yellowish. On DOI 9, dull headache started, which persisted for at least a week with gradual waning (Figure 2). The patient received no medication during the time course of the illness. On DOI 1, acute-phase blood was obtained from the patient for performing laboratory examinations. Laboratory examination results did not show significant finding except for a slightly elevated serum C-reactive protein level $(0.6 \mathrm{mg} / \mathrm{dL})$.

\section{Confirmation of RSV infection}

On DOI 1, throat swab specimen was obtained from the patient, and it was immediately inoculated to six kinds of cultured cells: HEp-2, Vero, MDCK, MNT-1, LLCMK2, and HFL-III. These cell lines are competent for isolating a variety of human respiratory viruses, including type A, B, and C influenza viruses; type 1, 2, 3, and 4 parainfluenza viruses; human metapneumovirus; RSV; mumps virus; herpes viruses; cytomegalovirus; adenoviruses; enteroviruses; and rhinoviruses (8). Six days after inoculation, the cytopathic effect (CPE) was recognized only in HEp-2 cells as a form of cell fusion that was characteristic of RSV infection and other cells exhibited no CPE. The positive and negative results of the CPE in these cells strongly suggested the exclusive isolation of RSV. Moreover, the clinical specimen and the supernatant of the culture for DOI 1 were used for RNA extraction. The RNA obtained from the culture supernatant was subjected to loop-mediated isothermal amplification (LAMP) assay, which has been established for detecting RSV (9). Using the LAMP assay, the RSV gene of subgroup B was detected, 


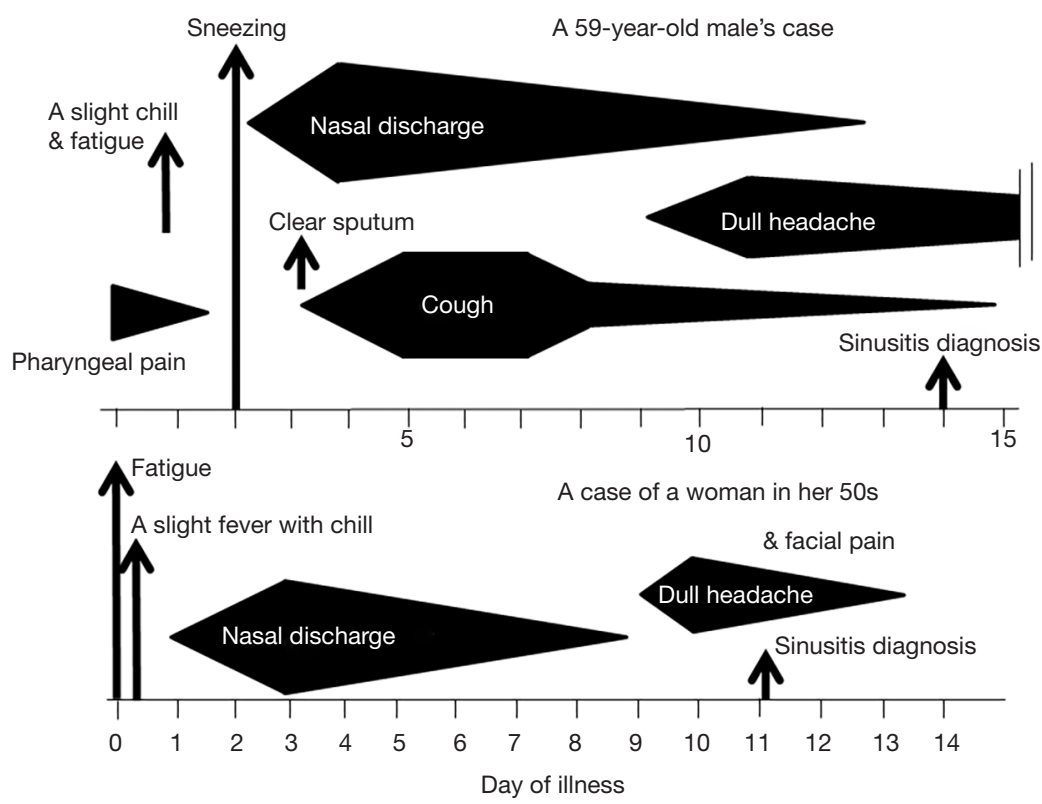

Figure 2 Time course analysis of symptoms of RSV infection. Schematic images of separately show the time courses of occurrence (arrows), duration, and strength of symptoms along the time after the onset of illness, for the two patients. The crescendo and decrescendo demonstrate the augmentation and remission of symptoms, respectively. RSV, respiratory syncytial virus.

genetically confirming RSV isolation. Finally, to exclude the possibility of co-infection with other possible pathogens, the extracted RNA from the clinical specimen was further subjected to multiplex real-time polymerase chain reaction (PCR), which can detect various respiratory pathogens with high sensitivity (10). We did not detect any genes of the pathogens listed in Table 1.

To serologically confirm RSV infection, the antibody against RSV was titrated for the paired sera of acute and convalescent phases, collected at DOI 1 and 3 weeks later, respectively. The titration was performed with the neutralization test using a contemporary strain of RSV subgroup B as the antigen, which was isolated in the same year of the RSV epidemic. We could not use the virus isolated from the subject himself because it did not grow well enough to be used for titration. In this assay, the neutralization titer was defined as the reciprocal of the highest dilution of the serum, at which infection with $100 \mathrm{TCID}_{50}$ of active virus in HEp-2 cells was perfectly blocked (11). The antibody titer showed an increase from 1:120 to 1:480, thereby confirming an RSV infection.

\section{Diagnosis of acute sinusitis}

On November 19, 2014, which was DOI 14 and 8 days after the onset of headache, the patient visited an outpatient brain surgery clinic, for an MR imaging (MRI) examination for pre-existent cervical spinal stenosis and then, also consulted the physician regarding his persisting headache. The physician observed thickening of the sinus mucosa and inferred that the headache could be a consequence of sinusitis. An otolaryngologist indicated the marked increase in mucosal thickness at the upper and middle areas of the right maxillary sinus and retention of mucus in the upper area (Figure $3 A, B)$. CT image taken after 6 months, i.e., May 14, 2015, during a dental treatment revealed that mucosal thickening disappeared (Figure 3C,D). Thus, the occurrence of acute sinusitis was proven in the MR and CT images of the sinus at acute and convalescent phases.

\section{Additional case}

The second case was a female in her 50s who also lived in Sendai City, Japan and who did not have without any health problems. Her illness started with fatigue on the morning of December 14, 2015 when RSV infection was spreading in the city (Figure 1). She had slight fever at noon, and her body temperature was approximately $37^{\circ} \mathrm{C}$ in the evening. However, she did not have pharyngeal pain. On the 
Table 1 Respiratory pathogens excluded

By culture only
Mumps virus
Herpes viruses: types 1,2
Cytomegalovirus
By real-time PCR analyses
Viruses
Influenza viruses: types A, B, C
Parainfluenza viruses: types $1,2,3,4 \mathrm{a}, 4 \mathrm{~b}$
Human metapneumovirus
Coronaviruses: $229 \mathrm{E}, \mathrm{OC} 43, \mathrm{NL} 63, \mathrm{HKU}$
Adenoviruses
Enteroviruses
Rhinoviruses
Bacteria
H. Influenza
Str. Pneumoniae
Sta. Aureus
B. Pertussis
Legionella
Others
Mycoplamydia
PR, Posis

$\mathrm{PCR}$, polymerase chain reaction.

following day, similar to the first case, rhinorrhea started and the amount of mucus discharge significantly increased, eventually becoming a runny nose on DOI 3 . Similar to the first case, RSV of subgroup B was isolated in HEp-2 cells among various cell line cells, which were inoculated with her nasal swab specimen of DOI 2. Moreover, only the RSV gene was detected in the specimen using multiplex real-time PCR (Table 1), as in the first case. The anti-RSV antibody was titrated for the paired sera with the neutralization test using the virus isolated from the patient as the antigen. The titers of acute and convalescent phase sera were 1:60 and $1: 240$, respectively, confirming RSV infection.

Her nasal discharge symptom waned within a week. However, the facial dull pain around the nasal area started on DOI 9 , and a clinical diagnosis of acute sinusitis was made by the physician. This pain symptom continued only for 4 days without receiving any medication. Unlike the first case, she did not have a symptom of coughing throughout her time course of illness (Figure 2).

\section{Discussion}

In this study, we presented cases of two healthy adults with RSV infections and describe their clinical data in detail from the early stage of the disease. This study unequivocally proved the occurrence of an RSV infection using virologically fundamental methods confirming elevated serum anti-RSV antibody titers, and also isolating active RSV. Detecting a pathogen using conventional PCR do not necessarily imply that the detected virus actually caused an infection, because it is occasionally a result of a false-positive reaction or a contamination without an infection. In addition, the detected pathogen may not be exclusively responsible for the illness, because sometimes more than one pathogen can be simultaneously detected in a sample that is examined using multiplex PCR $(12,13)$. We can reliably suggest that our cases solely had an RSV infection because negative results were obtained for various respiratory pathogens using multiplex PCR of their clinical specimens, which excluded the possibility of a coinfection with other pathogens.

Regarding the symptoms of RSV infection, pneumonia and bronchiolitis are not infrequent in children, particularly infants. The occurrence in children wanes as the age increases and reinfections repeatedly occur; the elderly and immune compromised individuals also sometimes suffer from lower respiratory inflammation (1). Clinical features of the infections in the adult population were reported in 204 patients diagnosed with RSV infection, although the diagnosis was based only on gene detection with conventional PCR for only RSV. Half of these cases were in their 50 's retrospectively and $>90 \%$ of them were reported to have symptoms of cough and nasal congestion, although the timings of symptoms were not known (14). Regarding definitive pure RSV infection in healthy adults, we found two reports of experimental infections in volunteers who were administered high amounts of attenuated RSV of a vaccine candidate strain via the nasal route. The subjects experienced symptoms of nasal stuffiness, sneezing, sore throat, or malaise, which persisted for 1-3 days (6); in addition, nasal discharge started on the second day and peaked on the seventh day after the inoculation (7).

Both of our cases did not have a symptom of nasal 

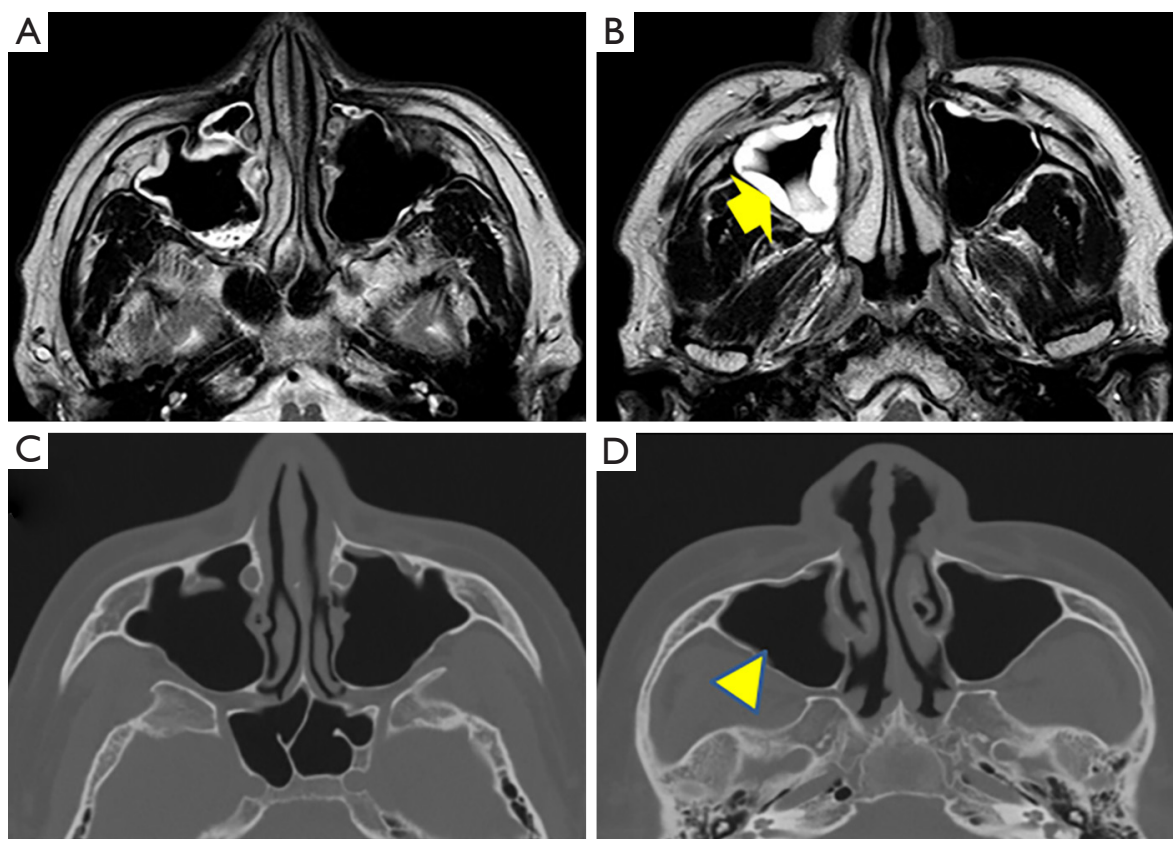

Figure $3 \mathrm{MRI}$ and CT images suggest acute sinusitis. (A,B) Axial views of the sinuses of the patient. The mucosal thickening was found to be increased in the MR images of the right maxillary sinus, slightly at the upper level (A) and significantly increased at the middle level (B, arrow); (C,D) CT image of the same levels after 6 months revealed that the mucosal thickening disappeared (arrow head). (C, upper level; D, middle level). MRI, magnetic resonance imaging; CT, computed tomography.

congestion but had rhinorrhea following slight fever; their general symptoms were only those of upper respiratory illness such as pharyngeal pain, sneezing and prolonged coughing. Furthermore, the dull headache or facial pain which was inferred as sinusitis, occurred approximately on DOI 9 in both cases. All of those symptoms were common with those previously reported (2).

Acute sinusitis itself is not infrequent and occurs in association with various acute upper respiratory infections with virus or bacteria $(15,16)$. In our first case, it is unclear whether sinusitis was caused by direct RSV infection to the site, or secondarily by other pathogen(s) such as bacteria following an RSV infection in the mucosa, which leads to the disturbance of airflows between the nasal cavity and sinus through the portal site. Sinusitis was diagnosed by CT images in 12 (63\%) among 19 immuno compromised adult patients with RSV pneumonia (17). Furthermore, sinusitis was clinically diagnosed by a physician in one of $12 \mathrm{RSV}$ infection cases, and four of 54 influenza cases (15). Examining whether RSV infection is prone to cause acute sinusitis would be a challenge for further studies, and future etiological studies of sinusitis associated with upper respiratory infections with many pathogen types will address this question.

\section{Acknowledgements}

We thank Dr. Shinsuke Suzuki (Department of Brain Surgery, Sendai Medical Center) for suggesting acute sinusitis during the patient's visit, Dr. Isolde Dapat and Enago (www.enago.jp) for English language review by a native speaker. This work was financially supported by the Clinical Research Division of Sendai Medical Center and the Research Program on Promoting Development of Innovative Drugs against Emerging and Reemerging Infectious Diseases from Japan Agency for Medical Research and Development, AMED.

\section{Footnote}

Conflicts of Interest: The authors have no conflicts of interest to declare.

Informed Consent: Written informed consent was obtained 
from the patients of this study.

\section{References}

1. Collins PL, Krron RA. Clinical features. Respiratory syncytial virus and metapneumovirus. In: Knipe DM, Howley PM, editors. Fields Virology, 6th edition. Philadelphia: Lippincott Williams-Wilkins, 2013;1086-123.

2. Hashem M, Hall CB. Respiratory syncytial virus in healthy adults: the cost of a cold. J Clin Virol 2003;27:14-21.

3. O'Shea MK, Ryan MA, Hawksworth AW, et al. Symptomatic respiratory syncytial virus infection in previously healthy young adults living in a crowded military environment. Clin Infect Dis 2005;41:311-7.

4. O'Shea MK, Pipkin C, Cane PA, et al. Respiratory syncytial virus: an important cause of acute respiratory illness among young adults undergoing military training. Influenza Other Respir Viruses 2007;1:193-7.

5. Park WJ, Yoo SJ, Lee SH, et al. Respiratory syncytial virus outbreak in the basic military training cAMP of the republic of Korea Air Force. J Prev Med Public Health 2015;48:10-7.

6. Hall CB, Douglas RG Jr, Schnabel KC, et al. Infectivity of respiratory syncytial virus by various routes of inoculation. Infect Immun 1981;33:779-83.

7. Buchman CA, Doyle WJ, Pilcher O, et al. Nasal and otologic effects of experimental respiratory syncytial virus infection in adults. Am J Otolaryngol 2002;23:70-5.

8. Numazaki Y, Oshima T, Ohmi A, et al. A microplate method for isolation of viruses from infants and children with acute respiratory infections. Microbiol Immunol 1987;31:1085-95.

Cite this article as: Nishimura H, Sato K, Kadji FM, Ohmiya S, Ito H, Kubo T, Hashimoto S. Case study-based time-course analysis of symptoms of respiratory syncytial virus infections followed by acute sinusitis in otherwise-healthy adults. J Thorac Dis 2018;10(5):E322-E327. doi: 10.21037/jtd.2018.04.74
9. Shirato K, Nishimura H, Saijo M, et al. Diagnosis of human respiratory syncytial virus infection using reverse transcription loop-mediated isothermal amplification. J Virol Methods 2007;139:78-84.

10. Enan KA, Nabeshima T, Kubo T, et al. Survey of causative agents for acute respiratory infections among patients in Khartoum-State, Sudan, 2010-2011. Virol J 2013;10:312.

11. Anderson LJ, Hierholzer JC, Bingham PG, et al. Microneutralization test for respiratory syncytial virus based on an enzyme immunoassay. J Clin Microbiol 1985;22:1050-2.

12. Scotta MC, Chakr VC, de Moura A, et al. Respiratory viral coinfection and disease severity in children: A systematic review and meta-analysis. J Clin Virol 2016;80:45-56.

13. Cebey-López M, Herberg J, Pardo-Seco J, et al. Viral Co-Infections in Pediatric Patients Hospitalized with Lower Tract Acute Respiratory Infections. PLoS One 2015;10:e0136526.

14. Sundaram ME, Meece JK, Sifakis F, et al. Medically attended respiratory syncytial virus infections in adults aged $\geq 50$ years: clinical characteristics and outcomes. Clin Infect Dis 2014;58:342-9.

15. Louie JK, Hacker JK, Gonzales R, et al. Characterization of viral agents causing acute respiratory infection in a San Francisco University Medical Center Clinic during the influenza season. Clin Infect Dis 2005;41:822-8.

16. Rosenfeld RM. CLINICAL PRACTICE. Acute Sinusitis in Adults. N Engl J Med 2016;375:962-70.

17. Mayer JL, Lehners N, Egerer G, et al. CT-morphological characterization of respiratory syncytial virus (RSV) pneumonia in immune-compromised adults. Rofo 2014;186:686-92. 